cases. The genetic data strongly implicates the endothelium as the initiating cell type in $\mathrm{PAH}$, and BMP9 is the major circulating ligand for BMPR-II on endothelial cells (EC). Since inflammation is emerging as a potential trigger for disease in BMPR-II mutation carriers, we hypothesised that BMP9 and BMPR-II mutation might impact on endothelial:leukocyte interaction.

Methods We employed blood outgrowth endothelial cells (BOEC) isolated from healthy donors (control-BOEC) and BOEC derived from PAH patients with BMPR-II mutations (PAH-BOEC) in this study.BOEC can be generated from venous blood and are recognised as a non-invasive source of patientderived EC. Endothelial:neutrophil interaction was studied using an in vitro flow-based model. Neutrophil recruitment to LPS (100 ng/ml; 4h)-stimulated BOECs was quantified in the presence or absence of BMP9 $(5 \mathrm{ng} / \mathrm{ml} ; 16 \mathrm{~h})$ under physiologically relevant sheer stress $(0.1 \mathrm{~Pa})$. Surface expression of E-selectin was quantified using flow cytometry, ELISA was used to assess the secretion of IL-8 and mRNA levels were quantified by qPCR.

Results LPS stimulation induced robust neutrophil recruitment to control-BOEC, whereas BMP9 had no effect.Co-stimulation with LPS and BMP9 resulted in a synergistic increase in neutrophil recruitment and control-BOEC E-selectin and IL-8 expression, compared to stimulation with LPS alone. Moreover, BMP9 stimulated the upregulation of TLR4 on control-BOEC (the receptor for LPS), thus providing a potential mechanism for the enhanced inflammatory response seen with LPS and BMP9 costimulation. In contrast to the findings in control-BOEC, LPS stimulation failed to induce neutrophil recruitment in $\mathrm{PAH}-$ BOEC. However, co-stimulation with LPS and BMP9 restored neutrophil recruitment in PAH-BOEC.

Conclusions BMP9 markedly enhances neutrophil recruitment to the endothelium in response to LPS. Deficiency in BMP9 signalling (as a consequence of BMPR-II mutations in PAH-BOEC) impairs this process, and BMP9 restores normal endothelial:neutrophil interactions in PAH-BOECs. These findings identify a hitherto unknown role for BMP9 in endothelial:neutrophil interaction and implicate this process in the pathobiology of PAH.

\section{S142 THE ROLE OF H3K27 METHYLATION IN VASCULAR ENDOTHELIAL CELL PROLIFERATION AND FUNCTION: IMPLICATIONS FOR PULMONARY ARTERIAL HYPERTENSION}

'D Shao, ${ }^{1} \mathrm{~N}$ Gambaryan, ${ }^{1} \mathrm{C}$ Meng, ${ }^{2} \mathrm{~F}$ Perros, ${ }^{2} \mathrm{M}$ Humbert, I Adcock, ${ }^{1} \mathrm{~S}$ Wort; ${ }^{1}$ Imperial College London, London, UK; ${ }^{2}$ Univ. Paris-Sud, Paris, France

\subsection{6/thoraxjnl-2013-204457.149}

Introduction and Objectives There is increasing interest in the role of epigenetic gene regulation in the pathogenesis of pulmonary arterial hypertension (PAH), a condition associated with pulmonary vascular cell proliferation. Methylation on histone H3K27 (H3K27me3) has been found to be a key regulator of development and cell homeostasis. Methylation at H3K27 can be reversed by Jumonji $\mathrm{C}(\mathrm{JmjC})$ domain-containing proteins, JMJD3. We were interested to determine the immunohistochemical distribution of $\mathrm{H} 3 \mathrm{~K} 27 \mathrm{me} 3$ and JMJD3 proteins in patients with IPAH and the effect of GSK-J4, an inhibitor of JMJD3, on markers of pulmonary vascular cell proliferation.

Methods Immunohistochemistry for JMJD3 and H3K27me3 was performed on human lung from normal healthy controls and patients with idiopathic PAH. The role of JMJD3 on cell cycle regulator (cyclin) expression in response to serum stimulation, with and without GSK-J4, was performed on normal human pulmonary artery endothelial cells (HPAECs) and RT-qPCR was then performed using QuantiTec primer assays. Chromatin Immunoprecipitation (CHiP) was performed antibody against H3K27me3 on normal HPAECs and analysed by quantitative PCR.

Results We found that the expression of JMJD3 protein is increased in the walls of pulmonary arteries of patients with idiopathic PAH compared to health control. This correlated to a decreased expression of H3K27me3. Treatment of HPAECs with GSK-J4, the inhibitor of JMJD3, in vitro, significantly increased cell cycle regulator p19, p27, CDK4 and CDK6 mRNA expression (Fig. $1, \mathrm{n}=3$ ), whilst having no effect on $\mathrm{p} 14, \mathrm{p} 16, \mathrm{p} 21$ and CDK2 (data not shown). Furthermore, CHiP using a specific H3K27me3 antibody, followed by RT-qPCR analysis, demonstrated that GSK-J4 treatment increased H3K27me3 occupation on the transcription start sites of p27 and CDK4, whilst the negative control compound GSK-J5 had no effect $(n=2)$.

Conclusion Our data suggest that JMJD3 and H3K27 histone methylation could play an important role in cell cycle progression and cell cycle exit of pulmonary vascular cells in patients with idiopathic PAH. As such drugs such as GSK-J4 may provide a therapeutic option in the future to reverse pulmonary vascular remodelling.

\section{S143 EVIDENCE THAT TYPE I INTERFERON DRIVES PULMONARY ARTERIAL HYPERTENSION}

${ }^{1} \mathrm{PM}$ George, ${ }^{1} \mathrm{E}$ Oliver, ${ }^{2} \mathrm{BE}$ Schreiber, ${ }^{2} \mathrm{AM}$ Holmes, ${ }^{3} \mathrm{M}$ Southwood, ${ }^{1} \mathrm{~S} J$ Wort, ${ }^{1} \mathrm{~N}$ Bartlett, ${ }^{4} \mathrm{P}$ Dorfmuller, ${ }^{3} \mathrm{NW}$ Morrell, ${ }^{2} \mathrm{G}$ Coghlan, ${ }^{4} \mathrm{M}$ Humbert, ${ }^{1} \mathrm{~L}$ Zhao, ${ }^{1} \mathrm{JA}$ Mitchell; ${ }^{1}$ Imperial College, London, UK; ${ }^{2}$ Royal Free Hospital, London, UK; ${ }^{3}$ Papworth Hospital NHS Trust, Cambridge, UK; ${ }^{4}$ Univ. Paris-Sud, Kremlin-Bic $\downarrow$ tre, France

\subsection{6/thoraxjnl-2013-204457.150}

Rationale There is increasing evidence of a link between interferon (IFN) and pulmonary arterial hypertension (PAH). Conditions such as HIV and Systemic sclerosis (SSc) where endogenous IFN levels are chronically elevated are strongly associated with $\mathrm{PAH}$. Furthermore, therapeutic use of type I IFN is now known to cause PAH and increase the systemic release of endothelin (ET)-1 in some patients. The link between IFN therapy and PAH was formally recognised at the 5-yearly World Symposium on Pulmonary Hypertension (Nice, Feb 2013). Our group has
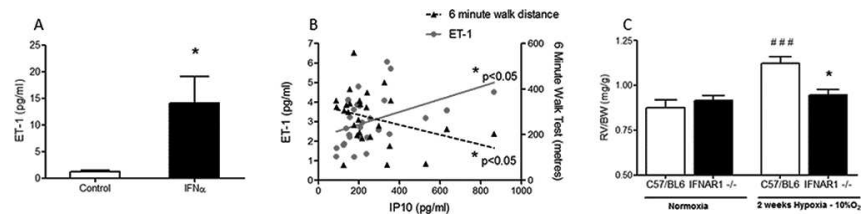

Abstract S143 Figure 1. A ET-1 release from human pulmonary artery smooth muscle cells. Data expressed as mean \pm SEM from $\mathrm{n}=\mathbf{5}$ experiments. Statistical significance determined by one-way ANOVA * $\mathbf{p}<0.05$. B Pearson Correlation between serum levels of IP10 and ET-1 (* $p<0.05)$ and IP10 and 6 minute walk test (* $\mathbf{p}<0.05)$ in 28 patients with Systemic Sclerosis associated PAH. C Mice lacking a functional type I IFN receptor (IFNAR1 $\%^{-}$) exposed to hypoxia $(10 \% \mathrm{O} 2)$ or normoxia compared to wild type (C57BI/ 6J) mice. Data presented as mean \pm SEM for $n=4-15$ mice. Right ventricular hypertrophy assessed as ratio of right ventricular (RV) mass to body weight (BW) (RV/BW). Statistical significance determined by one-way ANOVA followed by Bonferroni's multiple comparison post-test $(\# \# \# p<0.0001$ for normoxic vs. hypoxic conditions) and (*p $<0.05$ for IFNAR ${ }^{-} /$vs. $\mathrm{C} 57 \mathrm{BI} / 6 \mathrm{~J}$ mice). 


\section{Spoken sessions}

identified the mitogen ET-1 and the archetypal interferon stimulated gene - interferon gamma inducible protein 10 (IP10) as candidate mediators for IFN induced PAH. Using a comprehensive approach incorporating human pulmonary vascular cells in vitro, genetically modified mice in vivo and clinical samples with patient data we sought to explore the role of type I IFN in PAH. Methods Primary cells were cultured and responses measured using standard approaches. Serum cytokine and ET-1 levels were measured by ELISA. The role of type I IFN in PAH in vivo was determined using type I IFN receptor knock out (IFNAR1 ${ }^{-\%}$ ) mice and the chronic hypoxia model of PAH.

Results Human pulmonary artery smooth muscle cells expressed the type I IFN receptor and released ET-1 (Fig. 1a) and IP10 in response to IFN $\alpha$. In patients with SSc associated $\mathrm{PAH}$, serum
IP10 and ET-1 levels were raised and correlated positively together (Fig. 1b). In addition IP10 correlated strongly with pulmonary haemodynamics and 6 minute walk distance (Fig. 1b). Finally, IFN was found to mediate $\mathrm{PAH}$ in vivo since mice lacking a functioning type I IFN receptor were protected from hypoxia induced PAH (Fig. 1c).

Discussion and Conclusions These novel data reveal a clear pathological role for type I IFN in PAH and provide crucial insight into the mechanisms underpinning pulmonary vascular toxicity associated with type I IFN therapies. Furthermore, endogenous IFN elevated in autoimmune disease or viral infection, are now implicated in PAH. We conclude that type I IFN, via an action of IFNAR1 and potentially through ET-1 and IP10, mediates $\mathrm{PAH}$ and may represent a novel therapeutic target. 